\title{
Vegetación de un bosque de Polylepis incarum (Rosaceae) en el distrito de Lampa, Puno, Perú
}

\author{
Vegetation of a Polylepis incarum forest (Rosaceae) in Lampa district, Puno, Peru
}

\section{Daniel B. Montesinos-Tubée ${ }^{1,2,3}$, Ángel C. Pinto ${ }^{4}$, Diana F. Beltrán ${ }^{5}$ y Washington Galiano ${ }^{6}$}

\author{
1 Nature Conservation \& Plant Ecology Group. Wageningen University, The Netherlands. \\ 2 Naturalis Biodiversity Centre, Botany Section, National Herbarium of The Netherlands, Herbarium Vadense. Darwinweg 2, 2333 CR Leiden, The Netherlands. \\ 3 Instituto Científico Michael Owen Dillon, Arequipa, Perú. \\ 4 Facultad de Ecoturismo. Universidad Nacional San Antonio Abad del Cusco. Sede Quillabamba, Cusco, Perú. \\ 5 Asociación para el Desarrollo y la Investigación Suma Marka ONGD. Puno. \\ 6 Facultad de Ciencias Biológicas. Universidad Nacional de San Antonio Abad del Cusco, Perú. \\ Email Daniel Montesinos: dbmtperu@gmail.com \\ Email Ángel Pinto: pinto_angel@hotmail.com \\ Email Diana Beltrán: dfbeltranf@gmail.com \\ Email Washington Galiano: wgalianos2000@yahoo.es
}

\section{Resumen}

Se estudió la vegetación de los bosques de Polylepis incarum (Bitter) M. Kessler \& Schmidt-Lebuhn en el anexo de Lamparaquen, en la provincia de Lampa, departamento de Puno en los años 2009 y 2014. Se determinaron 83 especies vasculares pertenecientes a 35 familias. En cuanto a las formas de vida, dominan las herbáceas, gramíneas, arbustos y árboles, siendo en su mayoría, nativas e endémicas, algunas introducidas. Se identificó el ecosistema queñoal representado por la nueva unidad fitosociológica Lupino chlorolepis-Polylepidetum incari con cuatro subasociaciones respectivamente. La composición florística demuestra una riqueza moderadaalta de especies, siendo las familias con mayor representatividad, Asteraceae, Poaceae, Caryophyllaceae, Fabaceae y Pteridaceae. Se aplicó el método fitosociológico de Braun-Blanquet para el análisis de unidades vegetativas. Se presenta la clasificación de comunidades mediante el software TWINSPAN, el análisis de ordinación DCA con el software CANOCO 4.5 y un dendrograma basado en correlación de especies para cada cuadrante efectuado con el software PC-ORD. Mayores estudios son aún necesarios para tener una visión más amplia de las comunidades de plantas en otras zonas geográficas, y evaluar como la vegetación puede verse afectadas con el cambio climático y acciones antrópicas.

Palabras clave: vegetación; fitosociología; queñual; Lampa; Puno.

\section{Abstract}

The vegetation of the Polylepis incarum (Bitter) M. Kessler \& Schmidt-LeBuhn forests was studied in the Lamparaquen annex in the province of Lampa, Puno department in 2009 and 2014. A total of 83 vascular species belonging to 35 families were determined. The lifeforms are represented by herbs, grasses, shrubs and trees, being mostly native and endemic species with some introduced. The queñoal ecosystem is represented by the new phytosociological unit Lupino chlorolepis-Polylepidetum incari with four subassociations identified. The floristic composition shows a moderate to high species richness, being the most representative families, Asteraceae, Poaceae, Caryophyllaceae, Fabaceae and Pteridaceae. The Braun-Blanquet phytosociological method was applied for the analysis of the vegetation units. The classification of communities was done by means of TWINSPAN software, DCA ordination analysis with CANOCO 4.5 software and a dendrogram based on the correlation of species for each quadrant which was performed with PC-ORD software. Further studies are still needed to have a broader view of the plant communities in other geographic areas, and evaluate how vegetation may be affected by climate change and human actions.

Keywords: vegetation; phytosociology; queñual; Lampa; Puno.

\section{Citación:}

Montesinos-Tubée D.B. , W. Galiano, D.F. Beltrán y A.C. Pinto. 2015 Vegetación de un bosque de Polylepis incarum (Rosaceae) en el distrito de Lampa, Puno, Perú. Revista peruana de biología 22(1): 087 - 096 (Abril 2015). doi: http://dx.doi.org/10.15381/rpb.v22i1.11125

Presentado: $\quad 30 / 04 / 2014$

Aceptado: $\quad$ 22/03/2015

Publicado online: 24/04/2015
Información sobre los autores:

DBMT y ACP: realizaron el diseño experimental y el muestreo fitosociológico; DBMT y DFB: analizaron los datos; DBMT: redactó el manuscrito; DBMT, WG, DFB y ACP: revisaron y aprobaron el manuscrito. Los autores no incurren en conflictos de intereses. 


\section{Introducción}

En la región Puno, existen pocos estudios que traten la flora y ecología de sus diversos ecosistemas, especialmente para la provincia de Lampa, lugar que posee una gran riqueza cultural, histórica y también biológica donde se pueden encontrar diversas especies categorizadas como amenazadas. En cuanto a estudios biológicos, Reynel (1988), Sánchez et al. (1999), Arteta et al. (2006) y Gutiérrez \& Canales (2012) presentan trabajos sobre la diversidad florística de diferentes regiones geográficas andinas en el departamento de Puno. Numerosas especies peruanas del género Polylepis se encuentran categorizadas por la IUCN (2013).

Los estudios fitosociológicos realizados en bosques de Polylepis en el sur peruano y noroeste boliviano son aún escasos, siendo los trabajos de Fernández Terrazas (1997), Galán de Mera et al. (2002, 2003), Luebert \& Gajardo (2005) y Navarro \& Maldonado (2005) importantes contribuciones al conocimiento de la vegetación asociada a estos bosques. Kessler $(1995,2006)$ hace referencia a la vegetación de los bosques de Polylepis, donde resalta la dominancia de especies arbustivas y herbáceas asociadas a ellas, siendo el caso sobre todo en zonas semihúmedas a áridas, donde la estructura de los bosques es relativamente abierta permitiendo el desarrollo de una densa cubierta herbácea (Kessler 2006) corroborando la co-existencia de gramíneas de los géneros Festuca, Calamagrostis, Stipa y Mublenbergia, asi como herbáceas o subarbustos de los géneros Achyrocline, Baccharis, Bidens, Senecio, Stevia, entre otros. Los roquedales húmedos son poco comunes en los bosques de Polylepis habiéndose encontrado vegetación propia de estos ambientes y directamente asociado a Polyleps incarum en Lampa, como es el caso de algunas especies de los géneros Ribes, Cystopteris, Stellaria, Polystichum, entre otros.

La taxonomía del género Polylepis R. \& P. es muy compleja (Kessler \& Schmidt-Lebuhn 2006) y se caracteriza por una polinización anemófila y por su frutos secos (Kessler 2006). Polylepis incluye 50 especies sudamericanas (Mendoza \& Cano 2011) con 17-19 especies en Perú (Kessler \& Schmidt-Lebuhn 2006, Mendoza \& Cano 2011), en su mayoría árboles de entre 5 y 10 metros de altura. En el caso de Polylepis incarum (Bitter) M. Kessler \& Schmidt-Lebuhn, su sinonimia corresponde a Polylepis besseri subsp. incarum (Bitter) M. Kessler y Polylepis incana subsp. incarum Bitter, siendo Polylepis incarum (Bitter) M. Kessler \& Schmidt-Lebuhn el nombre aceptado (Kessler \& Schmidt-Lebuhn 2006). La distribución de Polylepis incarum se restringe para Bolivia y partes del departamento de Puno (Kessler \& Schmidt-Lebuhn 2006).

Las condiciones ambientales llevan a afirmar que el calentamiento global está causando un cambio gradual en la presencia de especies nativas y endémicas favoreciendo la rápida adaptación de diversas especies invasoras, causando desbalances en las comunidades biológicas (Pauli et al., 2007). Ciertjacks et al. (2008) presenta un importante estudio sobre la dinámica de los bosques de Polylepis y su degradación ante las condiciones de fuego. Los bosques de Polylepis incarum en la provincia de Lampa están sufriendo una fuerte influencia, degradación y aislamiento de sus bosques por la constante acción antrópica a través de la incesante quema para la obtención de áreas de cultivo, obtención de carbón, uso de leña o por incendios accidentales. Una evaluación preliminar ha demostrado la fuerte degradación que sufre tanto la queñua como las especies silvestres asociadas dando como resultado la invasión de especies ruderales e introducidas, afectando gravemente los ecosistemas de Polylepis y por consiguiente causando fragmentación y una dramática disminución de sus poblaciones. Sin embargo, información más detallada de las consecuencias de la quema en los varios estadios de Polylepis es aún desconocida (Ciertjacks et al. 2008, Kessler 2000).

Los objetivos del presente estudio fueron: a) estudiar la composición de la flora vascular de los bosques de Polylepis incarum en la Provincia de Lampa (departamento de Puno), b) conocer la estructura, composición y clasificación de las unidades fitosociológicas con Polylepis para la provincia de Lampa y c) elaborar un diagnóstico sobre la vulnerabilidad de los bosques de Polylepis incarum frente a la quema de sus ecosistemas.

\section{Materiales y métodos}

Área de estudio.- La localidad estudiada de Lamparaquen (distrito y provincia de Lampa), departamento de Puno, entre los $15^{\circ} 19^{\prime} 46^{\prime \prime}$ y $15^{\circ} 18^{\prime} 03^{\prime}$ 'S, $70^{\circ} 27^{\prime} 13^{\prime \prime}$ y $70^{\circ} 26^{\prime} 34^{\prime \prime W, ~ a ~} 3950$ - 4200 m. (Fig. 1). El área está caracterizada por sus grandes extensiones de planicies altiplánicas y laderas rocosas, humedales y terrenos agrícolas. La designación de cuadrantes de la vegetación se realizó en laderas rocosas de la zona denominada Lamparaquen, a $11 \mathrm{~km}$ al noroeste de Lampa durante junio (2009) y marzo (2014). La temporada de lluvias se extiende desde el mes de diciembre hasta el mes de abril. Durante el verano el clima es nublado, lluvioso y templado, mientras que durante el invierno el clima es frío y seco. En cuanto a la orografía, el río Lampa atraviesa las planicies altoandinas y algunas quebradas con humedad continua, otras temporalmente húmedas. En la región se practica la agricultura a base de maíz, habas, trigo, papa, olluco, oca, entre otros y la ganadería con ganado vacuno, ovino y auquénido.

Composición florística.- Para determinar la diversidad de especies vasculares se realizó una búsqueda completa en el área de muestreo que correspondió a laderas rocosas en ambos flancos del río Lampa. La determinación botánica de los taxones se realizó mediante revisión de herbario, uso de claves taxonómicas y consulta de las diferentes publicaciones disponibles en la literatura, tomando en consideración el trabajo de Brako \& Zarucchi (1993) y León et al (2008) y las versiones digitales de Missouri Botanical Garden y Field Museum of Natural History. Las consultas de herbario fueron realizadas, en su mayoría, en el herbario USM de la Universidad Nacional Mayor de San Marcos en Lima, el herbario HUSA de la Universidad Nacional de San Agustín y el herbario CUZ de la Universidad Nacional San Antonio Abad del Cusco.

Fitosociología, clasificación y análisis de datos.- Los cuadrantes fueron seleccionados de acuerdo a la homogeneidad de la vegetación y utilizando la metodología de ZürichMontpellier School of Phytosociology (Braun Blanquet, 1979), aplicándose el concepto de área mínima, basando el tamaño de las parcelas según la densidad de vegetación, tratándose de parches arbóreos con pajonales, los cuadrantes fueron establecidos con una dimensión de $225 \mathrm{~m}^{2}$ basando el tamańo aplicado para vegetación de tipo arbórea-arbustiva con pajonales según Kent \& Coker (1992). El muestreo de los cuadrantes fue realizado en junio 2009 y marzo 2014. Un total de 16 cuadrantes 

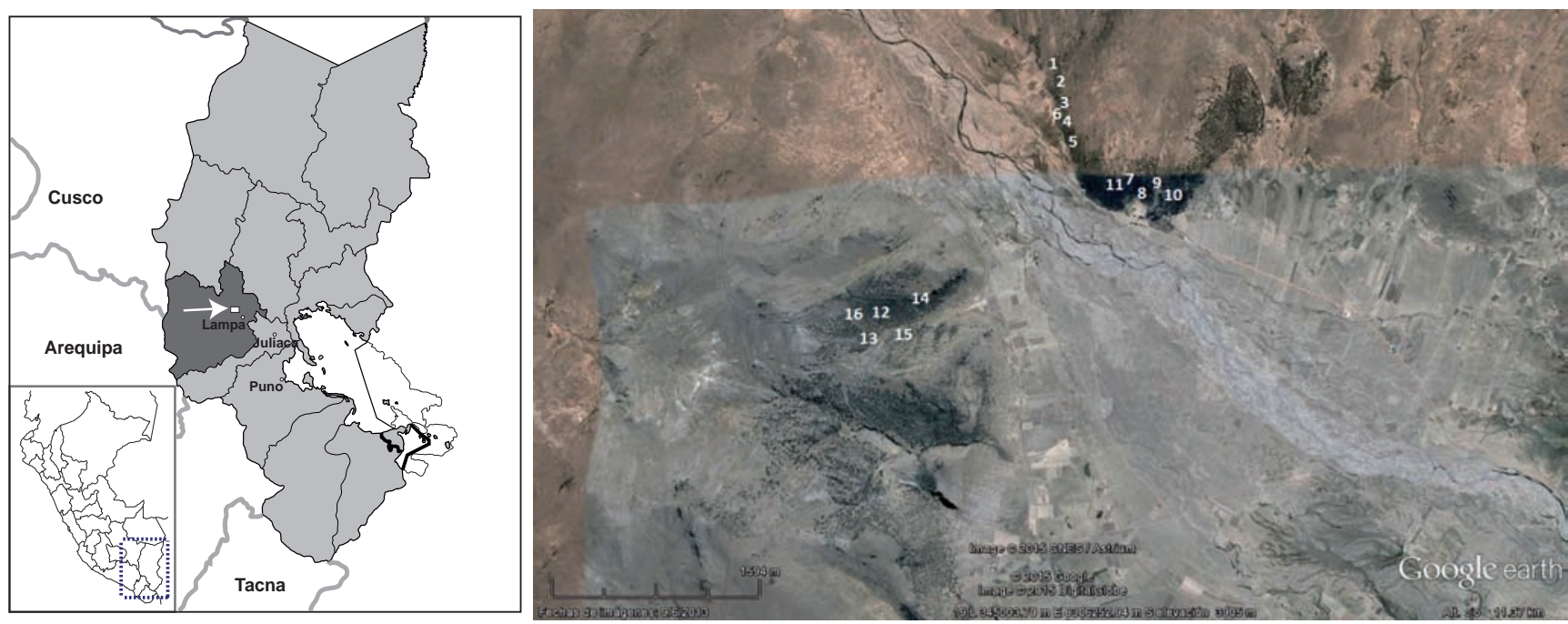

Figura 1. Mapa de ubicación de la localidad Figura 2. Ubicación de los cuadrantes estudiados. Provincia de Lampa, departaestudiada, provincia de Lampa, departa- mento de Puno. Fuente: "Lampa, Puno" 15¹8’49" S y 70²6’36" W. Google Earth. mento de Puno. Setiembre 4, 2013. Marzo 25, 2015.

fueron realizados en un área aproximada de $6 \mathrm{~km}^{2}$ en las laderas rocosas contiguas a ambos márgenes del río Lampa (Fig. 2). Para cada cuadrante, la presencia de especies fue anotada y la cobertura en campo fue estimada en porcentajes (Knapp 1984). Para los cálculos computarizados los valores porcentuales fueron transformados en la escala de ordenación de nueve puntos de cobertura/abundancia (Westhoff \& Van der Maarel 1973). Las escalas aplicadas fueron las siguientes: $0-1 \%=1 ; 2 \%=2 ; 3 \%$ $=3 ; 4-7 \%=4 ; 8-20 \%=5 ; 21-37=6 ; 38-68 \%=7 ; 69$ $-88 \%=8$ y $89-100 \%=9$.

Los nuevos sintaxones fueron descritos siguiendo el Código Internacional de Nomenclatura Fitosociológica (Weber et al. 2000); los cuadrantes fueron clasificados usando TWINSPAN (Hill 1979); los resultados sintaxonómicos fueron analizados utilizando un dendrograma con el test de promedio de correlación entre unidades con el software PC-ORD (McCune \& Mefford 1999). Las variables medioambientales (altitud, porcentaje de carbón y materia orgánica (restos vegetales), porcentaje de piedras y rocas y cobertura vegetal) fueron clasificados en una matriz cuadrante/especie y analizados mediante el Análisis Híbrido de Correspondencia sin tendencia (DCCA) con el software CANOCO 4.5 (Ter Braak \& Šmilauer 2002).

\section{Resultados}

Florística.- Se registraron un total de 83 especies, divididas en 72 géneros y 35 familias. Los pteridófitos están representados por dos especies y las monocotiledóneas con 9 especies. Las dicotiledóneas fueron el grupo mejor representado, con 83 especies, distribuidas en 52 géneros y 23 familias (Tabla 1). Las familias con mayor riqueza (Tabla 2) son Asteraceae con 18 géneros y 22 especies, seguido de Poaceae con 7 géneros y 7 especies, Caryophylláceas con 5 géneros y 7 especies, Fabáceas con cinco géneros y 6 especies y finalmente, Pteridaceae con 3 géneros y 4 especies. Un total de diez géneros poseen 2 especies cada una, el resto, 62, están representados por una especie. La familia Asteraceae, representa el 26.5\% de la flora total, mientras que Caryophyllaceae y Poaceae, representan en 8.4\% cada una, Fabaceae el $7.2 \%$ y Pteridaceae con el $4.8 \%$.
En cuanto a las formas de crecimiento, los helechos están representados por ocho especies (9.6\%), las suculentas con 3 especies $(3.6 \%)$, gramíneas con 6 especies $(7.2 \%)$, hierbas 45 especies $(54.2 \%)$, arbustos 19 especies $(23 \%)$ y árboles con 2 especies $(2.4 \%)$.

\section{Esquema Sintaxonómico}

Polylepidetea Tarapacano-Besseri Rivas Martínez \& Navarro 2002

Polylepidetalia Racemosae Galán de Mera \& Cáceres 2002

+ Ribesido brachybothrys-Polylepidion rugulosae Galán de Mera, Rosa \& Cáceres 2002, nom. mut. hoc loco [Ribesido brachybothrys-Polylepidion bessero Galán de Mera, Rosa \& Cáceres, Acta Bot. Malacit. 27: 87 (2002)].

Tabla 1. Divisiones taxonómicas con mayor riqueza florística (F: familias, G: géneros y $\mathrm{E}$ : especies) para los bosques de Polylepis incarum en Lamparaquen, Lampa, Puno.

\begin{tabular}{lccc}
\hline & F & G & SP \\
\hline Pteridophyta & 4 & 7 & 8 \\
Gimnospermas & 1 & 1 & 1 \\
Monocotiledóneas & 7 & 12 & 12 \\
Dicotiledóneas & 23 & 52 & 62 \\
\hline \multicolumn{1}{c}{ TOTAL } & 35 & 72 & 83 \\
\hline
\end{tabular}

Tabla 2. Familias con mayor riqueza florística para este estudio (G: géneros y E: especies) y el porcentaje de presencia a nivel de especies sobre el total de flora hallada.

\begin{tabular}{lccc}
\hline & G & SP & $\%$ \\
\hline Asteraceae & 18 & 22 & 26.5 \\
Poaceae & 7 & 7 & 8.4 \\
Caryophyllaceae & 5 & 7 & 8.4 \\
Fabaceae & 5 & 6 & 7.2 \\
Pteridaceae & 3 & 4 & 4.8 \\
\hline
\end{tabular}


Lupino chlorolepis-Polylepidetum incari Montesinos, Pinto, Beltrán \& Galiano associatio nova

poetosum candamoanae subassociatio nova

vicietosum graminae subassociatio nova

solanoetosum nitidae subassociatio nova

muehelenbeckietosum volcanicae subassociatio nova

\section{Descripción de comunidades}

Polylepidetea Tarapacano-Besseri Rivas Martínez \& Navarro 2002

\section{Polylepidetalia Racemosae Galán de Mera \& Cáceres 2002}

Queñuales del sur del Perú, Chile y Bolivia. Se caracterizan por la presencia de hierbas y arbustos propiamente andinos.

+ Ribesido brachybothrys-Polylepidion rugulosae Galán de Mera, Rosa \& Cáceres 2002, nom. mut. hoc loco [Ribesido brachybothrys-Polylepidion bessero Galán de Mera, Rosa \& Cáceres, Acta Bot. Malacit. 27: 87 (2002)]

\section{Queñuales del sur de Perú}

\section{Lupino chlorolepis-Polylepidetum incari Mon- tesinos, Pinto, Beltrán \& Galiano associatio nova}

Holotipo: Cuad. No. 5. Tabla 3. Figuras 3-5.

Fisionomía: Bosque perennifolio con altura media del dosel de unos 4-6 m en estado óptimo, y con baja diversidad de arbustos y relativa alta abundancia de hierbas anuales. El estrato arbóreo está dominado por Polylepis incarum (35 $40 \%$ de cobertura) y en menor densidad, Ribes brachybothrys con 3\% de cobertura. La densidad de arbustos (80 - 150 $\mathrm{cm}, 5-10 \%$ de cobertura) está caracterizada y dominada por Ageratina azangaroensis, Lupinus chlorolepis, Salpichroa hirsuta y Viguiera pazensis. La alta densidad de gramíneas está representada por Stipa ichu (8 - 10\% de cobertura) y Festuca dolichophylla $(3-4 \%)$. El estrato herbáceo, de $1-50 \mathrm{~cm}$ de altura, está representado por hierbas anuales, tanto cespitosas y arrosetadas, como erectas y algunas sufrútices, con una cobertura promedio de $30 \%$ y constituidos principalmente por Arenaria serpens, Bartsia crenoloba, Bidens andicola, Bowlesia lobata, Cardionema ramosissimum, Hieracium microcephalum, Hypochaeris chillensis, Lepechinia meyenii, Oxalis pachyrrhiza, Siphocampylus tupaeformis y Vicia graminea.

Sintaxonomía: La descripción de esta asociación se basa en 16 cuadrantes conteniendo 83 especies vasculares. Especies diagnósticas son: Hieracium microcephalum, Lupinus chlorolepis, Mutisia hastata, Oxalis pachyrrhiza, Polylepis incarum, Siphocampylus tupaeformis y Viguiera pazensis.

Ecología y distribución: Constituye el bosque de laderas montañosas del piso supratropical subhúmedo de las planicies altiplánicas y laderas rocosas adyacentes en la cuenca del río Lampa, en la provincia de Lampa, departamento de Puno. La asociación Lupino chlorolepis-Polylepidetum incari está distribuida a $3950-4200 \mathrm{~m}$. La vegetación se extiende sobre laderas rocosas con una inclinación que varía entre desde los $10^{\circ}$ hasta los casi $80^{\circ}$. Los suelos varían en la profundidad $(5-30 \mathrm{~cm})$ y su textura es predominantemente arcillo-limoso.

\section{poetosum candamoanae subassociatio nova}

Holotipo: Cuad. No. 1. Tabla 3.

Fisionomía: Bosque perennifolio localizado en pendientes muy rocosas, inclinadas y con afluentes de agua, ocasionales o permanentes. La vegetación es abundante y compuesta de hierbas, gramíneas, arbustos y árboles. Polylepis incarum alcanza una cobertura de $37 \%$. Característico es la presencia de numerosas hierbas y helechos propios de ambientes húmedos, como $A a$ mathewsii, Adiantum poiretii, Cystopteris fragilis, Oxalis nubigena, Peperomia peruviana, Polypodium pycnocarpum, Stellaria cuspidata, Stellaria weddellii y Woodsia montevidensis, que suman un 4-5\% de cobertura total. Arbustos como Ageratina azangaroensis, Mutisia hastata y Ophryosporus piquerioides son comunes, así como Ribes brachybothrys que alcanza una cobertura de $3 \%$. Stipa ichu está representado por el $8 \%$ de la cobertura total.

Sintaxonomía: La subasociación poetosum candamoanae se define en base a dos cuadrantes conteniendo 49 especies vasculares. A continuación se mencionan las especies diagnósticas: Aa mathewsii, Luzula racemosa, Oxalis nubigena, Poa candamoana y Stellaria cuspidata. Especies diferenciales de las demás subasociaciones: Adiantum poiretii, Asplenium peruvianum, Bomarea involucrosa, Cystopteris fragilis, Mutisia hastata, Ribes brachybothrys y Tillandsia capillaris.

Ecología y distribución: La subasociación se distribuye en laderas rocosas muy pronunciadas y continuamente húmedas, ubicadas en el margen izquierdo del río Lampa, en Lamparaquen, a una altitud de $3960 \mathrm{~m}$.

\section{vicietosum graminae subassociatio nova}

Holotipo: Cuad. No. 5. Tabla 3.

Fisionomía: Bosque perennifolio localizado en pendientes muy rocosas, inclinadas y bajo la cobertura continua de Polylepis incarum con 35 - 40\%. La dominancia de Lupinus chlorolepis y Vicia graminea son características. La cobertura total del área varía entre 37 y 58\%. La subasociación vicietosum graminae posee intervención antrópica habiéndose encontrado carbón (de P. incarum) por lo que algunas especies introducidas co-existen. Característico es encontrar alta frecuencia de Bidens andicola, Bowlesia lobata, Galinsoga quadriratiata, Festuca dolichophylla, Siphocampylus tupaeformis y Stipa ichu.

Sintaxonomía: La subasociación está basada en 4 cuadrantes con un total 56 especies vasculares. Diagnosticas son: Olsynium junceum, Ophryosporus piequerioides, Paranephelius ovatus y Vicia graminea.

Ecología y distribución: La subasociación se distribuye en laderas rocosas algo suaves y con signos de quema en las laderas. Se distribuye entre los 3940 y 4000 m en Lamparaquen, margen izquierdo del río Lampa. Estas quebradas fueron incidenaradas dos años antes del análisis de campo.

\section{solanoetosum nitidae subassociatio nova}

\section{Holotipo: Cuad. No. 10. Tabla 3.}

Fisionomía: Bosque perennifolio con alto grado de intervención humana. Se desarrolla en las laderas suaves adyacentes a los campos agrícolas. Numerosas especies ruderales pueden 


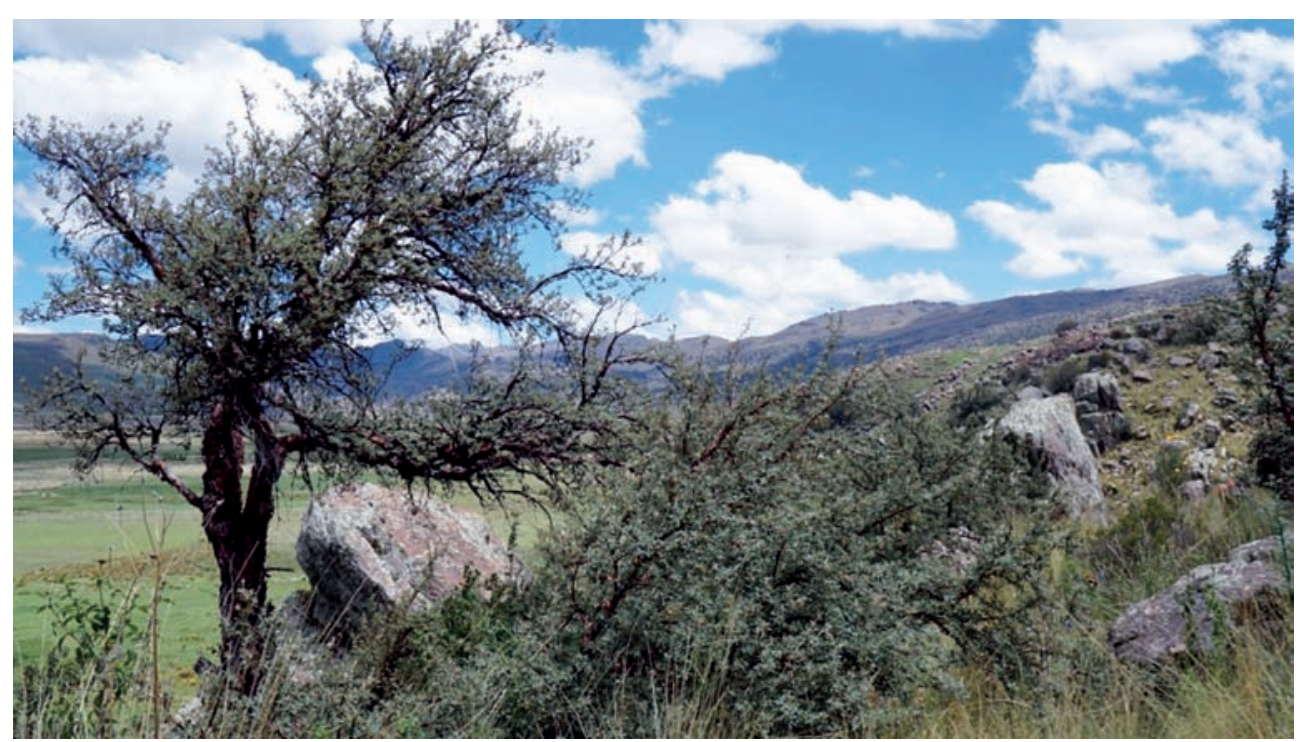

Figura 3. Polylepis incarum en su hábitat en Lamparaquen a $4000 \mathrm{~m}$.

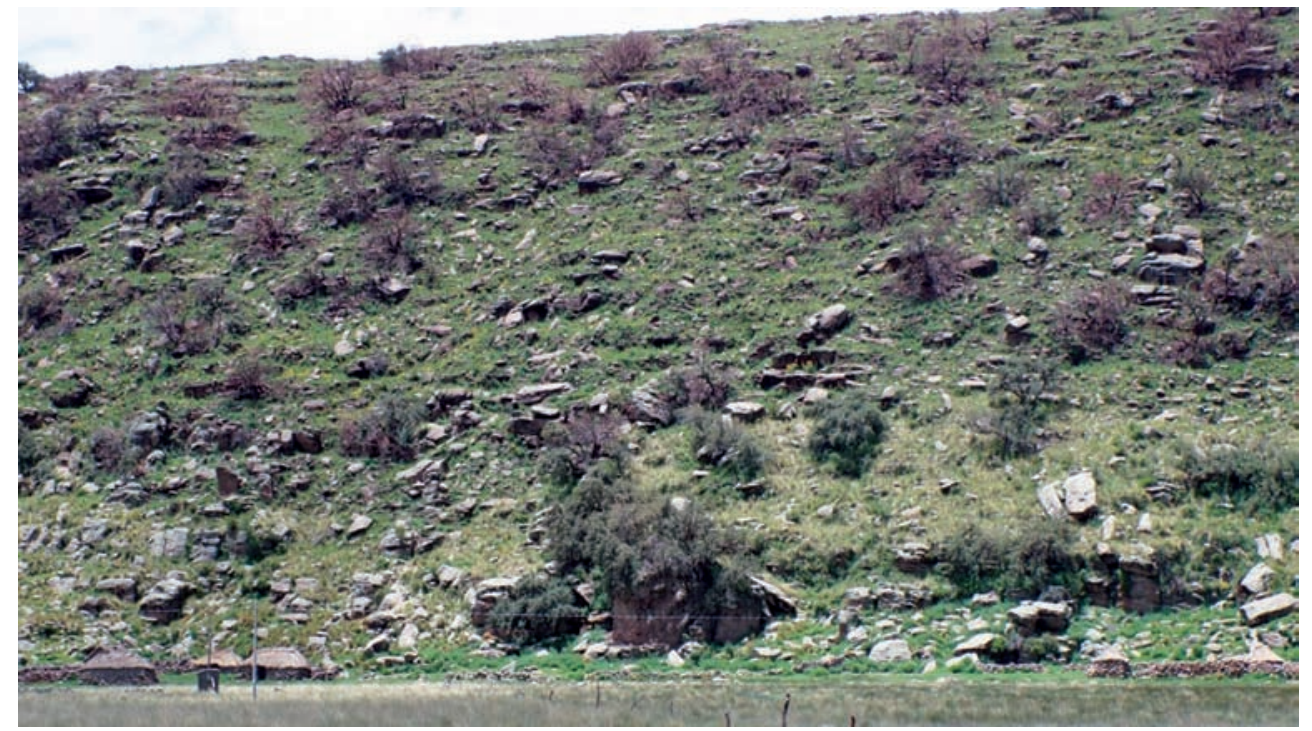

Figura 4. Bosque intervenido y bajo la influencia de fuego en Lamparaquen, a $3900 \mathrm{~m}$.

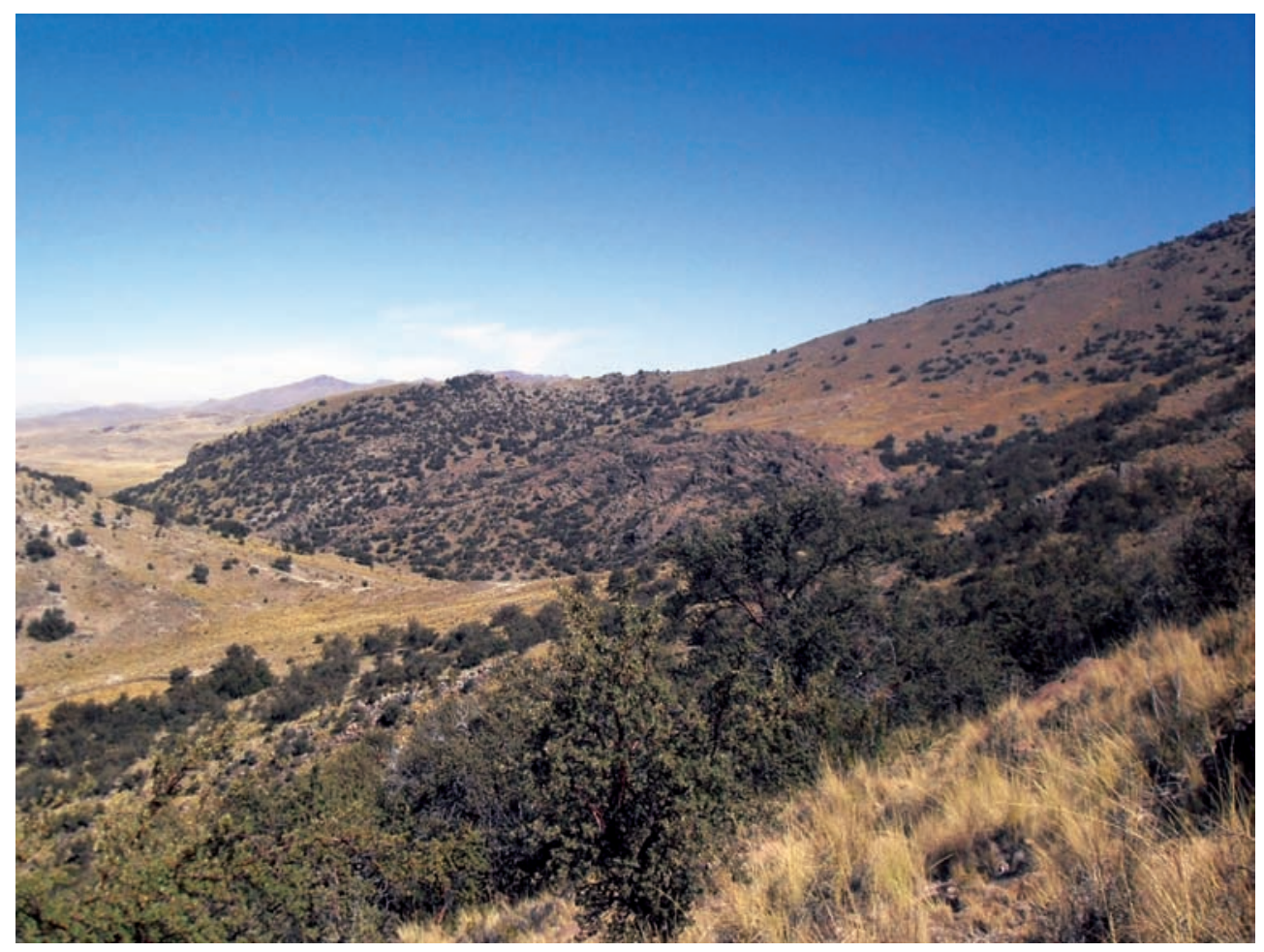

Figura 5. Bosque de Polylepis incarum donde se realizaron los cuadrantes fitosociológicos en Lamparaquen, provincia de Lampa, 4000 m. 
Tabla 3. Asociación de Lupino chlorolepis-Polylepidetum incari con sus respectivas subasociaciones. Para las ubicaciones de los cuadrantes referir a Figura 2.

\begin{tabular}{|c|c|c|c|c|c|c|c|c|c|c|c|c|c|c|c|c|}
\hline Cuadrantes & $\underline{1}$ & 2 & 3 & 4 & $\underline{5}$ & 6 & 7 & 8 & 9 & $\underline{10}$ & 11 & 12 & $\underline{13}$ & 14 & 15 & 16 \\
\hline Área & 225 & 225 & 225 & 225 & 225 & 225 & 225 & 225 & 225 & 225 & 225 & 225 & 225 & 225 & 225 & 225 \\
\hline Altitud & 3960 & 3970 & 3950 & 3955 & 3960 & 3940 & 4050 & 4060 & 4070 & 4070 & 4080 & 4200 & 4190 & 4190 & 4200 & 4180 \\
\hline$\%$ rocas & 40 & 50 & 20 & 15 & 22 & 20 & 15 & 18 & 18 & 15 & 20 & 25 & 20 & 12 & 20 & 15 \\
\hline$\%$ piedras & 10 & 10 & 15 & 12 & 10 & 12 & 20 & 22 & 22 & 25 & 20 & 12 & 14 & 15 & 15 & 12 \\
\hline \% carbón & 0 & 0 & 2 & 4 & 3 & 2 & 0 & 0 & 0 & 0 & 0 & 0 & 0 & 0 & 0 & 0 \\
\hline$\% \mathrm{MO}$ & 2 & 4 & 8 & 8 & 9 & 10 & 6 & 5 & 6 & 8 & 8 & 8 & 6 & 6 & 6 & 8 \\
\hline $\mathrm{CV}$ & 92 & 85 & 73 & 98 & 88 & 86 & 60 & 73 & 64 & 66 & 64 & 67 & 76 & 83 & 82 & 79 \\
\hline \#SPP & 38 & 35 & 33 & 22 & 34 & 29 & 15 & 19 & 15 & 15 & 14 & 16 & 25 & 21 & 27 & 28 \\
\hline
\end{tabular}

\section{Lupino chlorolepis-Polylepidetum incarum}

\begin{tabular}{|c|c|c|c|c|c|c|c|c|c|c|c|c|c|c|c|c|}
\hline Polylepis incarum & 7 & 6 & 6 & 6 & 7 & 7 & 6 & 7 & 6 & 7 & 7 & 7 & 6 & 7 & 6 & 6 \\
\hline Lupinus chlorolepis & 1 & & 3 & 2 & 2 & 2 & & & & 1 & & 1 & 2 & & & 1 \\
\hline Stipa ichu & 5 & 5 & 5 & 5 & 5 & 5 & 5 & 5 & 5 & 5 & 5 & 4 & 5 & 4 & 4 & 5 \\
\hline Festuca dolichophylla & 2 & & 3 & 3 & 4 & 3 & & 4 & 4 & & & & 4 & 4 & & \\
\hline Stevia macbridei & & 1 & 2 & & 1 & & & 1 & & & & & 1 & 1 & & \\
\hline Ageratina azangaroensis & 1 & 2 & & 1 & 2 & 1 & & 1 & & 1 & & 1 & & 1 & 1 & 1 \\
\hline Oxalis pachyrrhiza & & & 1 & & 2 & 1 & & & & 1 & & 1 & 1 & 1 & 1 & 1 \\
\hline Hieracium microcephalum & & 1 & & & 1 & 1 & & & & & & 1 & 2 & 1 & 1 & 1 \\
\hline Viguiera pazensis & 2 & & 2 & & 2 & 2 & 2 & & 2 & 2 & 1 & & & & & \\
\hline Siphocampylus tupaeformis & 1 & 1 & 2 & 2 & 2 & 1 & 1 & 2 & 2 & 1 & 2 & & & & & \\
\hline Mutisia hastata & 1 & 1 & & & & & & & & & & & & & 2 & 2 \\
\hline Ribes brachybothrys & 3 & 3 & & & & 2 & & & & & & & & 3 & 3 & 3 \\
\hline Bomarea involucrosa & 1 & 1 & & & & & & & & & & & & & 2 & 2 \\
\hline
\end{tabular}

\section{1 poetosum candamoanae}

\begin{tabular}{l|rr|l|l|l}
\hline Poa candamoana & 1 & 2 & & 1 & \\
Stellaria cuspidata & 1 & 2 & & \\
Oxalis nubigena & 1 & 1 & & \\
Luzula racemosa & 1 & 1 & & \\
Aa mathewsii & 1 & & & \\
\hline
\end{tabular}

\section{2 vicietosum graminae}

Ophryosporus piquerioides

Vicia graminea

Olsynium junceum

Paranephelius ovatus

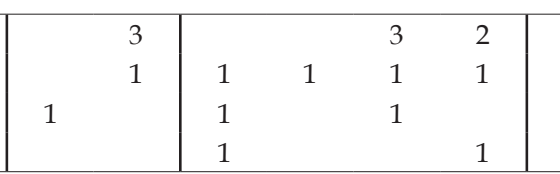

\section{3 solanoetosum nitidum}

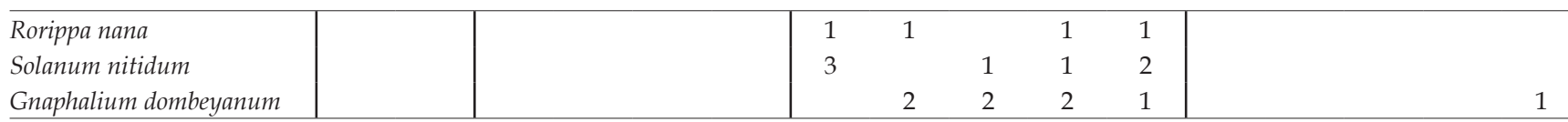

\section{4 muehelenbeckietosum volcanicae}

\begin{tabular}{|c|c|c|c|c|c|c|}
\hline Muehlenbeckia volcanica & & 2 & 2 & 2 & 1 & 1 \\
\hline Ephedra rupestris & 1 & 2 & 3 & 2 & & 1 \\
\hline Adesmia spinosissima & & 3 & 3 & 3 & 4 & 2 \\
\hline Senecio herrerae & & 1 & & & 1 & 1 \\
\hline Chuquiraga spinosa & & 2 & 3 & 2 & 3 & 3 \\
\hline Calamagrostis amoena & & & & & 2 & 2 \\
\hline
\end{tabular}

\section{POLYLEPIDETEA TARAPACANO-BESSERI Rivas Martinez \& Navarro 2002}

\section{POLYLEPIDETALIA RACEMOSAE Galán de Mera \& Cáceres 2002}

Ribesido brachybothrys-Polylepidion rugulosae Galán de Mera, Rosa \& Cáceres 2002

\section{ESPECIES DIFERENCIALES}

Caiophora cirsiifolia

Adiantum poiretii

Cystopteris fragilis

Woodsia montevidensis

Solanum sp.

Polypodium pycnocarpum

$\left|\begin{array}{ll|ll|}2 & & & \\ 1 & 1 & & \\ 1 & 1 & & 1 \\ 1 & 1 & 1 & \\ & 2 & 1 & \\ 1 & 1 & 1 & \end{array}\right|$


Tabla 3. (Continuacón ...)

\begin{tabular}{|c|c|c|c|c|c|c|c|c|c|c|c|c|c|c|c|c|}
\hline Peperomia peruviana & 1 & 1 & 1 & & & & & & & & & & & & & \\
\hline Hedeoma mandoniana & & 1 & 1 & & & & & & & & & & & & & \\
\hline Lobivia maximiliana & & & 2 & & & & & & & & & & & & & \\
\hline Cumulopuntia ignescens & & & 1 & & & & & & & & & & & & & \\
\hline Tarasa urbaniana & & & 2 & 2 & 2 & & & & & & & & & & & \\
\hline Galinsoga quadriradiata & & & 1 & 1 & 1 & 1 & & & & & & & & & & \\
\hline $\begin{array}{l}\text { Ranunculus praemorsus var. } \\
\text { praemorsus }\end{array}$ & & & & & 1 & 1 & & & & & & & & & & \\
\hline Plantago sp. & & & & 1 & 1 & & & & & & & & & & & \\
\hline Paronychia muschleri & & & & 1 & 1 & 1 & & & & & & & & & & \\
\hline Paronychia mandoniana & & & & & 1 & & & & & & & & & & & \\
\hline Medicago lupulina & & & & 1 & 1 & & & & & & & & & & & \\
\hline Gamochaeta americana & & & & 1 & & 1 & & & & & & & & & & \\
\hline Hypochaeris meyeniana & 1 & & 1 & & 1 & & & & & & & & & & & \\
\hline Geranium sessiliflorum & 1 & & 1 & & 1 & & & & & & & & & & & \\
\hline Cyperus seslerioides & 1 & 1 & 1 & & & 1 & & & & & & & & & & \\
\hline Lachemilla pinnata & 1 & & & & 1 & 1 & & & & & & & & & & \\
\hline Cerastium glomeratum & & 1 & & 1 & 1 & & & & & & & & & & & \\
\hline Stellaria weddellii & 1 & & & & & 1 & & & & & & & & & & \\
\hline Nassella pubiflora & 2 & & & & & 2 & & & & & & & & & & \\
\hline Gomphrena meyeniana & & 1 & & & 1 & & & & & & & & & & & \\
\hline Bidens andicola & 2 & 2 & 1 & 1 & 1 & 2 & & & & & & & & & & \\
\hline Hypochaeris chillensis & 1 & 1 & 1 & 1 & & 1 & & & & & & & & & & \\
\hline Galium hypocarpium & 1 & 1 & 1 & & 1 & 1 & & & & & & & & & & \\
\hline Astragalus micranthellus & & & & & & & 1 & 1 & 2 & 1 & 1 & & & 1 & 1 & \\
\hline Erigeron pazensis & & & & & & & 1 & & 1 & & & & & & 1 & 1 \\
\hline Achyrocline alata & & & & & & & & 1 & & & 1 & & 1 & 1 & & \\
\hline Quinchamalium procumbens & & & & & & & & 1 & & & 1 & & & & 1 & 1 \\
\hline Chersodoma jodopappa & & & & & & & & & & 3 & 3 & & & 3 & 3 & 3 \\
\hline Argyrochosma nivea & & & & & & & & 1 & & 1 & 1 & & & & 1 & 1 \\
\hline Baccharis caespitosa & & & & & & & 1 & 1 & 1 & & & 1 & 1 & 2 & 2 & \\
\hline Astragalus garbancillo & & & & & & & & 1 & 1 & & 1 & & 1 & & & \\
\hline Caiophora rosulata & & & & & & & & & & & & & 1 & & 1 & 2 \\
\hline Gnaphalium sp. & & & & & & & & & & & & & 1 & & & \\
\hline Senecio ferreyrae & & & & & & & & & & & & & 2 & 2 & & \\
\hline COMPAÑERAS & & & & & & & & & & & & & & & & \\
\hline Bartsia sp. & 1 & & 2 & & 2 & & 2 & & 2 & 1 & & & 2 & & & \\
\hline Muhlenbergia peruviana & & 1 & 1 & & 1 & & 1 & 1 & & & 1 & & & & 1 & 1 \\
\hline Cheilanthes pruinata & 1 & 1 & 1 & 1 & & 1 & 1 & & 1 & 1 & & 1 & 1 & & & 1 \\
\hline Cardionema ramosissimum & 1 & & 1 & 1 & & 1 & 1 & 1 & 1 & & & & 1 & & 1 & 1 \\
\hline Bowlesia lobata & & 1 & 1 & 1 & 1 & 1 & & 1 & 1 & & & & 1 & 1 & 1 & \\
\hline Salpichroa hirsuta & 1 & 1 & & 1 & & & & & & & & & 2 & 1 & 2 & 2 \\
\hline Asplenium peruvianum & 1 & 1 & & & & & & & & 1 & & 1 & 1 & 1 & 1 & 1 \\
\hline Lepechinia meyenii & 1 & & 1 & 2 & 2 & & & & & & & 2 & 2 & & & 2 \\
\hline Cheilanthes pilosa & & 1 & & & 1 & & & & & & & & 1 & 1 & 1 & \\
\hline Arenaria serpens & & & 1 & & 1 & & 1 & & & 1 & & 1 & & & 1 & 1 \\
\hline Baccharis tricuneata & & & & & 2 & 2 & & & & & & & & & & \\
\hline Tillandsia capillaris & 1 & 1 & & & & & & & & & & & & & & \\
\hline
\end{tabular}




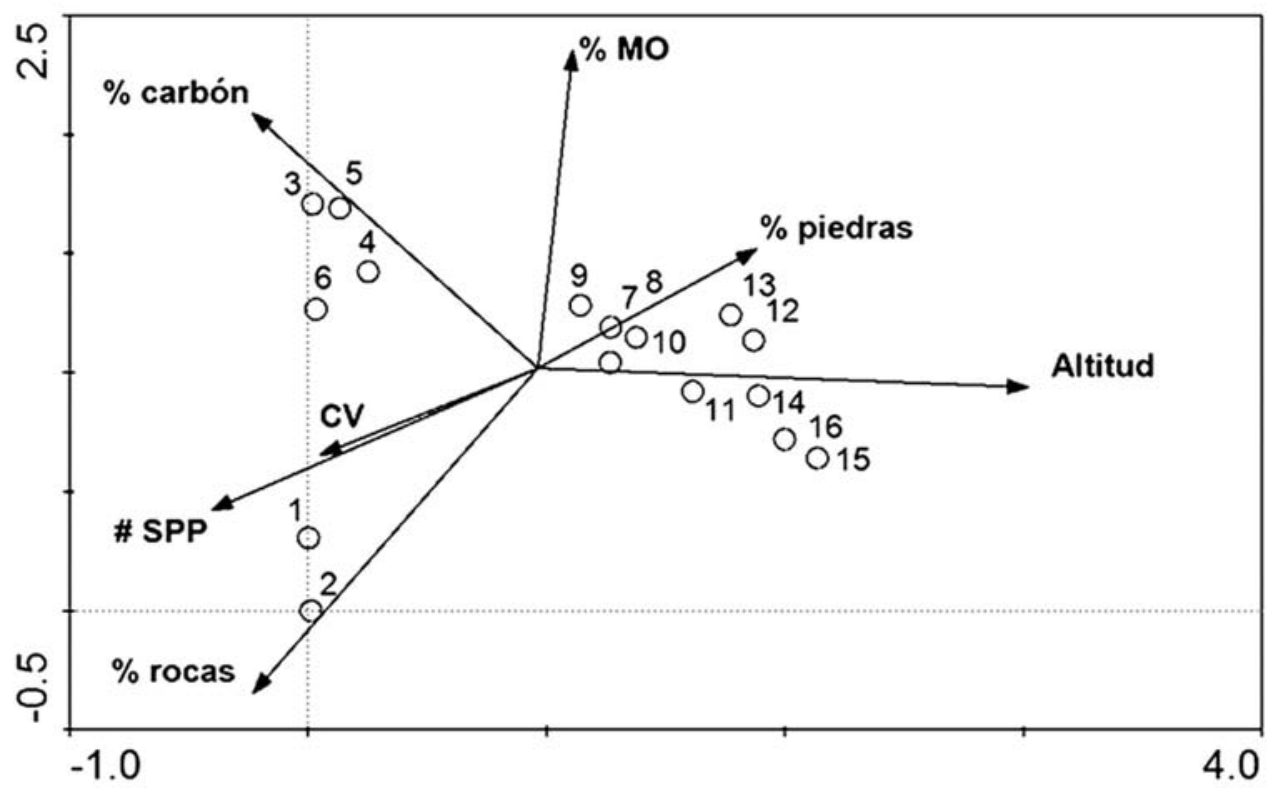

Figura 6. Análisis de DCA con diferentes variables medioambientales en los bosques de Polylepis incarum en la provincia de Lampa. Referir a Tabla 3 para identificar la ubicación de los números de cuadrantes.

encontrarse en este tipo de bosques. La cobertura vegetal es relativamente alta, con $80-100 \%$. Polylepis incarum alcanza $40 \%$ de cobertura y Stipa ichu con $8-10 \%$ de cobertura. Característica es la elevada frecuencia de especies como Gnaphalium dombeyanum, Siphocampylus tupaeformis, Solanum nitidum y Viguiera pazensis.

Sintaxonomía: La subasociación está basada en 5 cuadrantes con un total de 28 especies vasculares. Diagnóstico es encontrar a Gnaphalium dombeyanum, Rorippa nana y Solanum nitidum. Especies diferenciales con relativa alta frecuencia: Astragalus garbancillo, Astragalus micranthellus, Baccharis caespitosa y Chersodoma jodopappa.

Ecología y distribución: La subasociación se distribuye en laderas rocosas cercanas a los campos agrícolas y constituidos por una fuerte intervención humana. Se distribuye entre los 4050 y 4080 m en Lamparaquen, margen derecho del río Lampa.

\section{muehelenbeckietosum volcanicae subassociatio nova}

Holotipo: Cuad. No. 13. Tabla 3.

Fisionomía: Bosque perennifolio con pendientes suaves y suelo rocoso. La cobertura vegetal varía entre 70 y $80 \%$ y es relativamente alta, con $80-100 \%$. Polylepis incarum alcanza 35-40\% de cobertura y Stipa ichu con 6-8\% de cobertura. Característica es la elevada frecuencia de especies arbustivas como Adesmia spinosissima, Chuquiraga spinosa y Muehlenbeckia volcanica.

Sintaxonomía: La subasociación está basada en 5 cuadrantes con un total de 40 especies vasculares. Especies diagnósticas como Adesmia spinosissima, Calamagrostis amoena, Chuquiraga spinosa, Ephedra rupestris, Muehlenbeckia volcanica y Senecio herrerae.

Ecología y distribución: La subasociación se distribuye en laderas suaves y rocosas cerca del límite superior del bosque, entre los 4180 y 4200 m en el margen izquierdo del río Lampa, en
Lamparaquen. Nótese la existencia de numerosas ruinas y muros incas en la zona de análisis y que están densamente cubiertos por la vegetación.

\section{Análisis estadístico de ordenación y dendrograma}

El análisis de ordinación se presenta con un diagrama de DCA (Fig. 6) donde se puede apreciar la asociación Lupino chlorolepis-Polylepidetum incari (cuadrantes 1 - 16) donde los cuadrantes 1 y 2 representado por poetosum candamoanae que está correlacionado con porcentaje de rocas y mayor número de especies. Los cuadrantes $3-6$, representados por subasociación vicietosum graminae están fuertemente influenciados por porcentaje de carbón y cobertura vegetal. Por otro lado, los cuadrantes 7 - 16, representados por solanoetosum nitidum y muehelenbeckietosum volcanicae están correlacionados con altitud, porcentaje de materia orgánica y porcentaje de piedras.

El dendrograma (Fig. 7) se realizó utilizando la medida de distancia de Sorensen (Bray-Curtis) y el método de grupo por vinculación con valores promedio. Este método nos permitió identificar la separación de las subasociaciones de Lupino chlorolepis-Polylepidetum incari. L1-L2 están representados por poetosum candamoanae, L3-L6 por subasociación vicietosum graminae, L7-L11 por solanoetosum nitidum y finalmente L12L16 por muehelenbeckietosum volcanicae.

\section{Discusion}

La clase Polylepidetea tarapacano-besseri Rivas Martínez \& Navarro 2002, Galán de Mera et al. (2002, 2003) y Navarro \& Maldonado (2005) se caracteriza por ser comunidades de bosques de Queñua que se distribuyen desde Argentina hasta Ecuador. En tanto que Ribesido brachybothrys-Polylepidion bessero Galán de Mera, Rosa \& Cáceres 2002 (Galán de Mera et al. 2002, 2003) se distribuye en la puna seca del sur de Perú y noroeste de Bolivia. Las asociaciones que presentan esta alianza, dentro de las cuales se describe la nueva asociación Lupino chlorolepis-Polylepidetum incari, están caracterizadas por su distribución en regiones altiplánicas del centro del departamento 


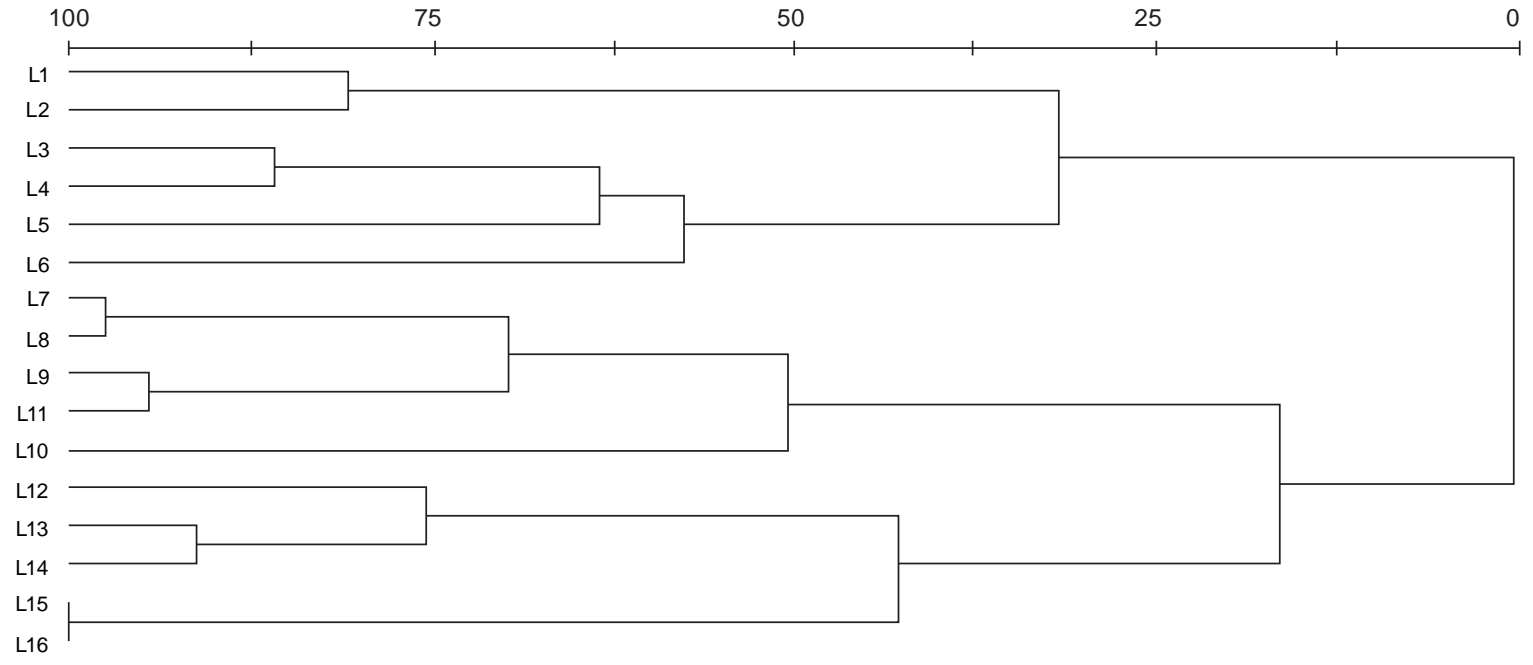

Figura 7. Dendrograma con las unidades fitosociológicas representadas por Polylepis incarum en la provincia de Lampa. Referir a Tabla 3 para identificar la ubicación de los números de cuadrantes.

de Puno donde la vegetación es típica de la puna y constituida por diversas especies arbustivas, herbáceas y algunas gramíneas. La diversidad florística en estos bosques es media-alta y se encuentran fuertemente influenciadas por el fuego ocasionado por la acción antrópica. Las poblaciones de queñua están disminuyendo cada vez más y hoy en día son escasos los lugares donde se encuentran bosques en buen estado. Se presume que Lampa posee uno de los refugios de bosques de queñua más importantes en el sur de Perú pero que lamentablemente corren el riesgo de la desaparición de sus ecosistemas por la tala y quema indiscriminada de los últimos tiempos.

\section{Conclusiones}

Concluimos que la unidad fitosociológica constituida por Lupino chlorolepis-Polylepidetum incari, se desarrolla en laderas rocosas en regiones altiplánicas del departamento de Puno. Poco menos de 6 especies endémicas se han encontrado y numerosas especies ruderales dan muestra del grado de intervención humana en este tipo de bosques. Se ha podido apreciar que numerosos parches boscosos han sufrido la quema total de sus ecosistemas durante la temporada seca, eventos que no se habían visto cuando se realizaron los análisis en el 2009. Se concluye que los bosques de Polylepis incarum están sufriendo una fuerte degradación y se corre el riesgo de la pérdida total o parcial de sus frágiles ecosistemas en un corto plazo. Es urgente establecer regímenes de conservación para preservar a futuro los bosques de Polylepis incarum en el departamento de Puno.

\section{Agradecimientos}

Los autores están agradecidos a Vilma Pacheco de Albaracin y Amanda Frisancho Camacho por facilitar el acceso a las zonas evaluadas y la hospitalidad brindada durante el trabajo de campo en Lampa. A los herbarios HUSA, USM y CUZ por la disponibilidad de sus colectas al momento de realizar las revisiones. Agradecemos a Antoine M. Cleef y Antonio Galán de Mera por los comentarios fitosociológicos. Asimismo agradecemos a Hamilton Beltrán, María Isabel La Torre y Víctor Quipuscoa S. por sus comentarios en la taxonomía de las especies.

\section{Literatura citada}

Arteta M., M. Corrales, C. Dávalos, A. Delgado, F. Sinca, L. Hernani \& J. Bojórquez. 2006. Plantas vasculares de la bahía de Juli, Lago Titicaca, Puno - Perú. Revista Ecología Aplicada 5(1-2): 29-36.

Brako L. \& J. Zarucchi. 1993. Catalogue of the Flowering Plants and Gymnosperms of Peru.- Monographs in Systematic Botany, Missouri Botanical Garden. 45: 1-1286.

Braun-Blanquet J. 1979. Plant sociology, the study of plant communities. - Transl. by G. D. Fueller and H. S. Conard. Mc. Graw-Hill, New York. 438 pp.

Cierjacks A., K. Weschel \& I. Hensen. 2008. Post-Fire Population Dynamics of Two Tree Species in High-Altitude Polylepis Forests of Central Ecuador. Biotropica 40(2): 176-182. DOI: http://dx.doi.org/10.1111/j.1744-7429.2007.00361.x

Fernández Terrazas E. 1997. Estudio fitosociológico de los bosques de Kewiña (Polylepis spp., Rosaceae) en la Cordillera de Cochabamba. Revista Boliviana de Ecología y Conservación Ambiental 2: 49-65.

Galán de Mera A., M.V. Rosa \& C. Cáceres. 2002. Una aproximación sobre la vegetación del Perú. Clases, órdenes y alianzas. Acta Botanica Malacitana 27: 75-103.

Galán de Mera A., C. Cáceres \& A. Gonzáles. 2003. La vegetación de la alta montańa andina del sur de Perú. Acta Botanica Malacitana 28: 121-147.

Gutiérrez I.R. \& A. Canales. 2012. Evaluación comparativa de la diversidad de flora silvestre entre la isla Taquile y el cerro Chiani en relación a la altitud, Puno, Perú. Revista Ecología Aplicada 11(2): 39-46

Hill M.O. 1979. Twinspan, a Fortran program for arranging multivariate data in an ordered two way table by classification of the individuals and the attributes. Cornell University, Department of Ecology and Systematics, Ithaca, New York.

IUCN. 2013. IUCN Red List of Threatened Species. Version 2013.2 www.iucnredlist.org. Accedido el 29 de Abril 2014.

Kent M \& P. Coker. 1992. Vegetation Description and Analysis. A Practical Approach. CRC Press, pp: 44-84.

Kessler M. \& A.N. Schmidt-Lebuhn. 2006. Taxonomical and distributional notes on Polylepis (Rosaceae). Organisms, Diversity \& Evolution 6, Electr. Suppl. 1: 1-10.

Kessler M. 1995. The genus Polylepis (Rosaceae) in Bolivia. Candollea 42: 31-71. 
Kessler M. 2000. Observations on a human-induced fire event at a humid timberline in the Bolivian Andes. Ecotropica 6: 89-93.

Kessler M. 2006. Bosques de Polylepis. En: Moraes M., B. Øllgaard, L.P. Kvist, F. Borchsenius \& H. Balslev (Eds.) Botánica Económica de los Andes Centrales. Universidad Mayor de San Andrés, La Paz. pp: 110-120.

Knapp R. 1984. Considerations on quantitative parameters and qualitative attributes in vegetation and phytosociological relevés. In: R. Knapp (Ed.). Sampling methods and taxon analysis in vegetation science (Handbook of Vegetation Science). Springer Netherlands. 77-100 pp.

León B., Roque, J., Ulloa, C., Pitman, N., Jorgensen, P.M. \& A. Cano. 2006. El Libro Rojo de las Plantas endémicas del Perú. Revista Peruana de Biología. Número Especial 13(2): 1-965.

Luebert F. \& R. Gajardo. 2005. Vegetación alto andina de Parinacota (norte de Chile) y una sinopsis de la vegetación de la Puna meridional. Phytocoenologia 35(1): 79-128. DOI: http:// dx.doi.org/10.1127/0340-269X/2005/0035-0079

McCune B. \& M.J. Mefford. 1999. PC-ORD for Windows. Multivariate Analysis of Ecological Data Version 4.25. MjM Software, Gleneden Beach, Oregon, U.S.A.

Mendoza W. \& A. Cano. 2011. Diversidad del género Polylepis (Rosaceae, Sanguisorbeae) en los Andes peruanos. Revista Peruana de Biología 18(2): 197-200. DOI: http://dx.doi. org/10.15381/rpb.v18i2.228

Navarro G. \& M. Maldonado. 2005. Geografía Ecológica de Bolivia.
Vegetación y Ambientes Acuáticos. Centro de Ecología Simón I. Cochabamba - Bolivia. 247-268, 454-499.

Pauli H., Gottfried M. \& K. Reiter. 2007. Signals of range expansions and contractions of vascular plants in the high Alps: observations (1994-2004) at the GLORIA*master site Schrankogel, Tyrol, Austria. Global Change Biology 13: 147-156, DOI: http://dx.doi.org/10.1111/j.1365-2486.2006.01282.x.

Reynel C. 1988. Plantas para Leña en el Sur-Occidente de Puno. Proyecto Arbolandino Puno - Perú. 1-100.

Sánchez F., Vicente A., Cruz J., Sánchez J., Durán A. \& U. Quispe. 1999. Biodiversidad de plantas silvestres a diferentes altitudes en la quebrada del cerro de Huaje, Puno. ANCA S.A. Impresiones Puno - Perú. 48pp.

Ter Braak W. \& P. Šmilauer. 2002. CANOCO. Reference manual and CanoDraw for Windows User's Guide: Software for Canonical Community Ordination (version 4.5). Microcomputer Power, Ithaca, NY, USA.

Weber H.E., Moravec J. \& J.P. Theurillat. 2000. International Code of Phytosociological Nomenclature. 3rd edition. Journal of Vegetation Science 11: 739-768. DOI: http://dx.doi. org $/ 10.2307 / 3236580$

Westhoff V. \& E. Van der Maarel. 1973. The Braun-Blanquet approach.-In: Whittaker, R.H. (ed.): Handbook of Vegetation Science 5: 617-650. 